\title{
miR-4262 promotes the proliferation of human cutaneous malignant melanoma cells through KLF6-mediated EGFR inactivation and p21 upregulation
}

\author{
DINGWEI ZHANG, ZHANGJUN LI, YANFEI ZHANG, CHEN TU, JIA HUO and YAN LIU \\ Department of Dermatology, The Second Hospital of Xi'an Jiaotong University, Xi'an, Shaanxi 710004, P.R. China
}

Received February 21, 2016; Accepted April 8, 2016

DOI: $10.3892 /$ or.2016.5190

\begin{abstract}
Alterations in the levels and functions of microRNAs (miRs) have been associated with carcinogenesis. In this study, we investigated the role and underlying mechanism of miR-4262 in the proliferation of human cutaneous malignant melanoma (CMM) cells. The expression levels of miR-4262 were significantly upregulated in cancerous tissues compared with those in matched adjacent normal tissues from $110 \mathrm{CMM}$ patients. miR-4262 was also regulated in five types of CMM cell lines, displaying an opposite expression pattern to that of Kruppel-like 6 (KLF6), a proven tumor suppressor in several cancers other than CMM. KLF6 overexpression sharply reduced A375 cell proliferation, suppressed the activation of epidermal growth factor receptor (EGFR) and increased p21 expression levels, while knockdown of KLF6 by siRNA transfection had an opposite effect. Furthermore, KLF6 was proven to be a direct target gene of miR-4262 by bioinformatic analysis and KLF6-3'UTR luciferase reporter assay. Finally, our data on miR-4262 mimic and inhibitor transfection indicated that miR-4262 could markedly reduce the expression of KLF6 protein and had a stimulatory effect on A375 cell proliferation. Our findings indicate that KLF6 acts as a tumor suppressor in CMM cells and miR-4262 promotes the proliferation of CMM cells through KLF6-mediated EGFR inactivation and p21 upregulation.
\end{abstract}

\section{Introduction}

Cutaneous malignant melanoma (CMM) is a type of cancer that develops from melanocytes and includes four subtypes according to clinical organization criteria: malignant nevoid lentigo maligna melanoma (LMM), superficial spreading

Correspondence to: Dr Jia Huo, Department of Dermatology, The Second Hospital of Xi'an Jiaotong University, 157 Xiwu Road, Xi'an, Shaanxi 710004, P.R. China

E-mail: huojjaa@126.com

Key words: cutaneous malignant melanoma, microRNA-4262, proliferation, Kruppel-like 6 melanoma (SSM), acral lentiginous melanoma (ALM), and nodular melanoma (NM). Environmental factors, such as ultraviolet irradiation and family heredity, have been regarded as high-risk factors for the incidence of CMM (1). An increasing number of studies reveal that mutation or aberrant activation of certain signaling pathways also contributes significantly to the incidence and progression of CMM, including the anti-oncogene p53, the p21/cyclin D1/cyclin-dependent kinase 4 (CDK4) pathway, and the epidermal growth factor receptor (EGFR)/Akt pathway $(2,3)$.

The transcription factor Kruppel-like 6 (KLF6) is an antitumor member of the KLF zinc finger DNA-binding protein family that plays an important role in the regulation of cell proliferation, migration, and apoptosis (4). Inactivation and downregulation of KLF6 have been regarded as an important sign of tissue carcinogenesis (5). In many parenchymal carcinomas, KLF6 suppresses activation of the EGFR/Akt pathway or the p21-mediated expression of cyclin D1/CDK4, and functions as a tumor suppressor (6-8). However, its role and underlying mechanism in the regulation of malignant melanoma are not yet fully understood. Several recent studies demonstrated that the expression level of KLF6 in patients with malignant melanoma is significantly decreased, and its level is negatively correlated with the size and metastasis of the tumor (9-11). Thus, KLF6 is likely to play a negative regulatory role in the development of melanoma.

MicroRNAs (miRNAs) are involved in cancer initiation, progression, and treatment response. miR-4262 is a newly identified miRNA and a provisional family member predicted in human embryonic stem cells and neural precursors (12), the function of which has not been well studied. Recent research has shown that miR-4262 expression is aberrantly expressed in patients with prostate cancer (13). Another study on adenocarcinoma pathogenesis indicated that miR-4262 expression was dysregulated and had an effect on cisplatin resistance of adenocarcinoma cells (14). These clues suggest that miR-4262 may play a role in the regulation of cancer initiation or progression.

In this study, we found that miR-4262 was markedly upregulated in various types of human CMM tissues and cell lines, displaying an opposite expression pattern to that of KLF6. We then explored the roles of KLF6 and miR-4262 in the proliferation of CMM cell lines, as well as their interaction. 


\section{Materials and methods}

Ethics statement and sampling. The study enrolled $110 \mathrm{CMM}$ patients of stages I and II, including 30 LMM (mean age $45 \pm 9.2$ years), $30 \mathrm{SSM}$ (mean age $47 \pm 9.8$ years), 30 ALM (mean age $46 \pm 9.5$ years) and $20 \mathrm{NM}$ (mean age $45 \pm 9.4$ years) cases, with an equal number of males and females. The patients had no evidence of lymph node metastasis and their tumor diameters were $\leq 2 \mathrm{~cm}$. Moreover, none of the patients had received preoperative anticancer treatment. CMM tissues and matched adjacent normal epithelial tissues were obtained from each subject by minimally invasive surgery. The study was approved by the Ethics Committee of The Second Affiliated Hospital of Xi'an Jiao Tong University. The donors and their guardians were previously informed of the experimental details and provided written consents.

Cell culture and transfection. Normal human melanocytes HACAT and foreskin fibroblasts HFFs, as well as human CMM cell lines A375, Malme-3M, SK-MEL-2, SK-MEL-5, and $\mathrm{M} 14$ were cultured in a $5 \% \mathrm{CO}_{2}$ atmosphere at $37^{\circ} \mathrm{C}$ in Dulbecco's modified Eagle's medium (DMEM) with $10 \%$ fetal bovine serum (FBS), and $100 \mathrm{mg} / \mathrm{ml}$ penicillin and $100 \mathrm{U} / \mathrm{ml}$ streptomycin (all from Gibco, Rockville, MD, USA). All cell lines were purchased from the American Type Culture Collection ${ }^{\circledast}$ (ATCC; Rockefeller, MD, USA).

When the cell density reached approximately $70 \%$ confluency, the pcDNA-KLF6 expression vector, KLF6 siRNA, or the single-stranded oligo miRNA inhibitors or mimics (designed and synthesized by Gene Pharma, Shanghai, China) were transfected into the cells with Lipofectamine ${ }^{\circledR} 3000$ (Invitrogen, Carlsbad, CA, USA) according to the manufacturer's instructions.

3'-UTR luciferase reporter assay. The wild-type 3'-UTR of human KLF6 mRNA was amplified by PCR, and a truncated 3'-UTR with deletion of the miR-4262 targeting site was amplified by nested PCR, using primers linked with the XhoI and NotI restriction sites at the beginning and end, respectively. All primers were designed and synthesized by GenScript Co., Ltd. (Nanjing,China). The PCR products were excised with Not $\mathrm{I}$ and $\mathrm{XhoI}$ and inserted into the $\mathrm{psiCHECK}^{\mathrm{TM}}-2$ vector (Promega, Madison, WI, USA) at the $3^{\prime}$ end of the Renilla gene CDS. Firefly luciferase activity was used as the internal control. The WT/truncated 3'-UTR dual-luciferase vectors were transfected or co-transfected with the miR-4262 mimic into 293T cells (100\% confluence; ATCC) using X-tremeGENE 9 DNA transfection reagent (Roche, Basel, Switzerland). The medium was changed $6 \mathrm{~h}$ later. Cells were incubated for another $48 \mathrm{~h}$ with commercial cell lysis buffer (Merck \& Co., Inc., Whitehouse Station, NJ, USA). The luciferase activity was measured using a luminometer (Promega) according to the manufacturer's instructions.

Real-time qPCR. Total RNA of the tissues or cells was extracted with TRIzol reagent (Takara, Dalian, China) according to the manufacturer's instructions. After quality and integrity were checked, $\sim 1,000 \mathrm{ng}$ of total RNA was used in the first-strand cDNA synthesis reaction. The miR-4262 stem-loop primer and quantitative primers, as well as the pre-miR-4262 quantitative primers, were designed and produced by Invitrogen. Each individual sample was run in triplicate wells. PCR amplification cycles were performed using the iQTM5 Multicolor Real-Time PCR detection system (Bio-Rad Laboratories, Inc., Hercules, CA, USA) and SYBR Premix Ex Taq II kit (Invitrogen). The reactions were initially denatured at $95^{\circ} \mathrm{C}$ for $3 \mathrm{~min}$ followed by 40 cycles of $95^{\circ} \mathrm{C}$ for $10 \mathrm{sec}, 55^{\circ} \mathrm{C}$ for $30 \mathrm{sec}$, and $72^{\circ} \mathrm{C}$ for $20 \mathrm{sec}$. U6 RNA was used to normalize the expression of miR-4262, and $\beta$-actin was used to normalize the expression of other RNA transcripts. The $2^{-\Delta \Delta C t}$ method was used to evaluate the relative expression level of the RNA transcripts.

Western blotting. Cells were lysed in lysis buffer (Beyotime Institute of Biotechnology, Shanghai, China) containing $1 \mathrm{mM}$ PMSF. The concentration of total protein was determined using the BCA protein assay (Tiangen Biotech Co., Ltd., Beijing, China). Fifty micrograms of protein in each sample was separated by $12 \%$ SDS-PAGE and then transferred to PVDF membranes (Millipore, Boston, MA, USA) for immunoblotting analysis. The following primary antibodies were used: anti-KLF6 (1:300), anti-EGFR (1:400), anti-p21 (1:300), anti-cyclin D1 (1:300), anti-CDK4 (1:200) (Abcam, Cambridge, MA, USA), and anti-GAPDH (1:800; Santa Cruz Biotechnology, Inc., Santa Cruz, CA, USA), which was used as the internal reference. After incubation with the appropriate HRP-conjugated secondary antibody, the proteins were detected using a ChemiDoc XRS imaging system and analysis software Quantity One (Bio-Rad Laboratories, Inc.).

Detection of cell proliferation. The cell proliferation assay was performed using the CellTiter-Blue H cell viability assay kit (Promega) and the MTT method (Sigma-Aldrich, St. Louis, MO, USA) according to the manufacturer's instructions.

Online website. The targeting relationship between miR-4262 and KLF6 was evaluated by the online server TargetScan (http:// www.targetscan.org/cgi-bin/targetscan/vert_70/view_gene.gi?r $\mathrm{s}=$ ENST00000542957.1\&taxid $=9606 \&$ members $=\&$ showcnc $=$ $0 \&$ shownc $=0$ \&showncf\&subset $=1$ ).

Statistical analysis. Each experiment was carried out for a minimum of three independent replicates, and each replicate experiment was performed in triplicate. Data are represented as mean \pm SEM. Statistics were calculated using SPSS 19.0 (SPSS Inc., Chicago, IL, USA). Multiple comparisons were assessed by one-way ANOVA followed by Dunnett's tests. Differences between groups were considered statistically significant at $\mathrm{P}<0.05$.

\section{Results}

miR-4262 is upregulated in different types of human CMM tissues and multiple human CMM cell lines. To explore the potential role of miR-4262 in the progression of human CMM, the levels of miR-4262 were first examined in cancerous epithelial tissues and matched adjacent normal epithelium of the CMM patients, including $30 \mathrm{LMM}, 30$ SSM, 30 ALM, and $20 \mathrm{NM}$ patients. The results showed that miR-4262 expression was upregulated in all of the cancerous tissues compared with that noted in the matched tissues (Fig. 1A-D). Then, the 
A

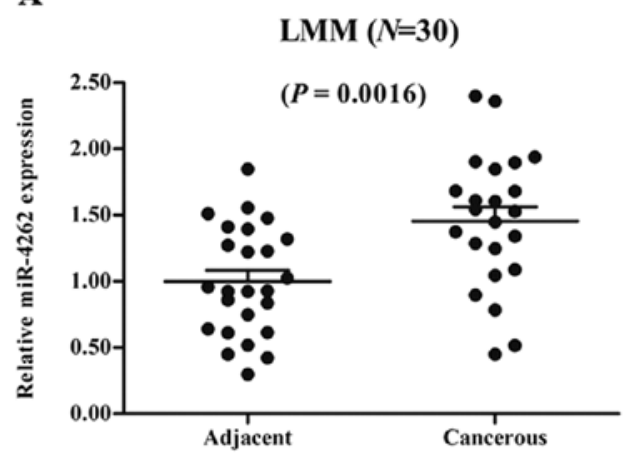

C

$\operatorname{ALM}(N=30)$

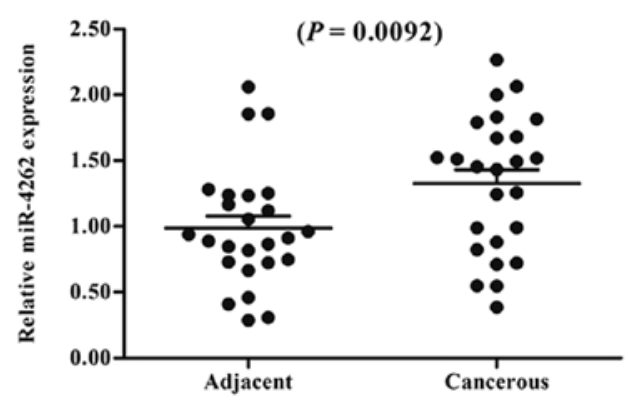

B

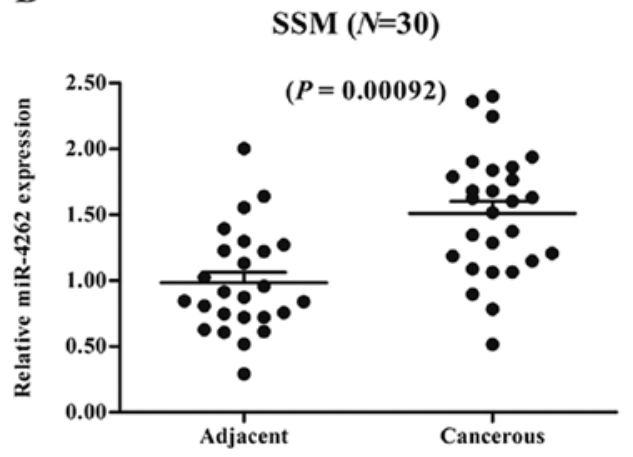

D

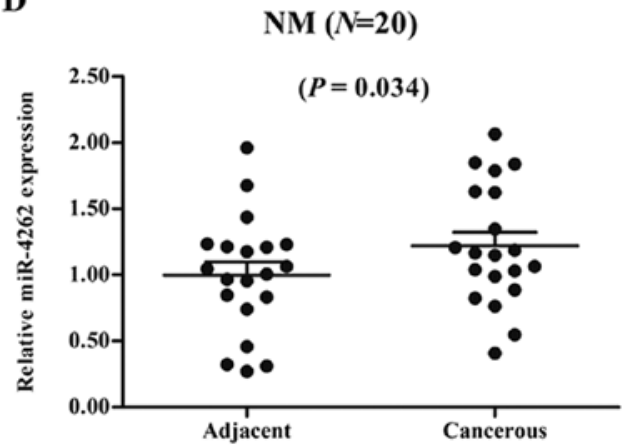

Figure 1. miR-4262 is upregulated in human cutaneous malignant melanoma tissues. (A) miR-4262 was upregulated in human LMM tissues compared with matched adjacent normal tissues. (B) miR-4262 was upregulated in human SSM tissues compared with matched adjacent normal tissues. (C) miR-4262 was upregulated in human ALM tissues compared with matched adjacent normal tissues. (D) miR-4262 was upregulated in human NM tissues compared with matched adjacent normal tissues. Cancerous epithelial tissues and matched adjacent normal epithelium were sampled from $110 \mathrm{CMM}$ patients, including 30 LMM, 30 SSM, 30 ALM and 20 NM patients. Total RNA was extracted from the samples and the expression levels of miR-4262 were detected with real-time qPCR. CMM, cutaneous malignant melanoma; LMM, lentigo maligna melanoma; SSM, superficial spreading melanoma; ALM, acral lentiginous melanoma; NM, nodular melanoma. Differences between the tissues were considered statistically significant at $\mathrm{P}<0.05$.

levels of pre-miR-4262 and miR-4262 were detected in two types of normal human melanocytes and five types of CMM cell lines. Our results showed that, compared with the normal melanocytes, pre-miR-4262 and miR-4262 were both robustly upregulated in the CMM cell lines (Fig. 2A and B). These data suggest that miR-4262 may be involved in the regulation of CMM progression.

KLF6 is identified as a target gene of $m i R-4262$. Recent studies indicate that the transcription factor KLF6 is downregulated in patients with malignant melanoma and negatively correlated with melanoma growth and metastasis. The expression levels of mRNA and protein were detected in the CMM cell lines in this study. Our results showed that KLF6 mRNA and protein were also significantly downregulated in the CMM cell lines (Fig. 2C and D), displaying an opposite expression pattern to that of miR-4262. Then the targeting relationship between miR-4262 and KLF6 was evaluated using the online server TargetScan. The output showed that the miR-4262 seed sequence completely matched bases $2955-2962$ of the KLF6 mRNA 3'-UTR (Fig. 3A). 3'-UTR luciferase reporter assay revealed that the miR-4262 mimic markedly reduced fluorescence intensity in the psiCHECK-WT 3'-UTR group but had no effect on that of the mutant 3'-UTR group (with a deletion of bases 390-420; Fig. 3B). Moreover, the miR-4262 mimic and inhibitor were respectively transfected into the CMM A375 cells, and western blotting showed that the miR-4262 mimic sharply reduced the expression of KLF6 protein while the miR-4262 inhibitor had an opposite effect (Fig. 3C). The above data demonstrated that miR-4262 directly targets and negatively regulates the expression of KLF6.

KLF6 suppresses the proliferation of A375 human CMM cells. To explore the exact role of KLF6 in the proliferation of CMM cells, a pcDNA-KLF6 expression vector and KLF6 siRNA were individually transfected into A375 human CMM cells. Cell proliferation was evaluated using the MTT and CellTiter-Blue $\mathrm{H}$ assays. The results showed that A375 cell proliferation was notably reduced by pcDNA-KLF6 transfection and increased by the KLF6 siRNA transfection (Fig. 4A and B). Simultaneously, KLF6 overexpression significantly reduced the level of the pro-tumorigenic protein EGFR, increased that of the tumor suppressor p21, and caused a decrease in the levels of cyclin D1 and CDK4 (Fig. 4C). KLF6 siRNA transfection had an opposite effect on the expression of the above proteins when compared to the results from KLF6 overexpression (Fig. 4C). These data indicate that KLF6 has a negative effect on CMM cell proliferation.

miR-4262 suppresses KLF6 expression and promotes the proliferation of A375 cells. Finally, the role of miR-4262 in the proliferation of CMM cells was explored. A singlestranded oligo miR-4262 mimic and inhibitor were transfected individually into the A375 cells. Our data from the MTT and 
A

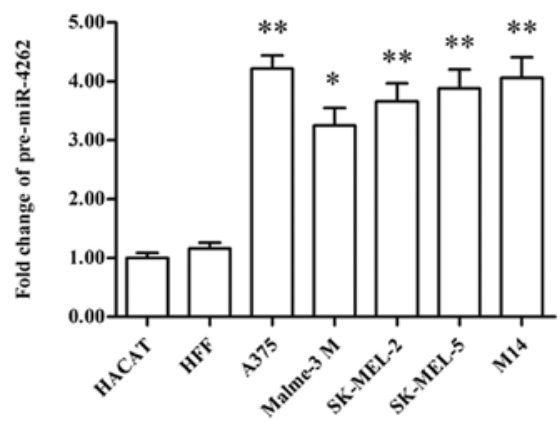

C

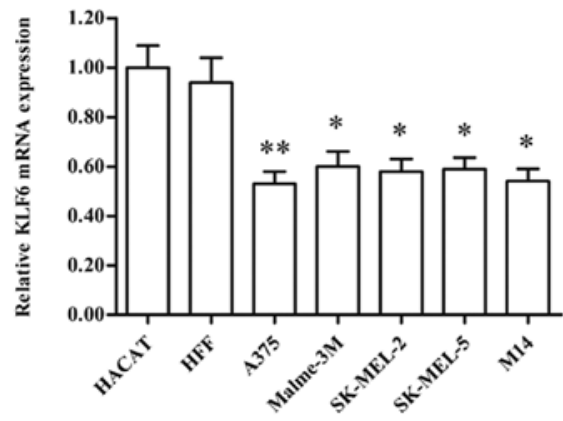

B

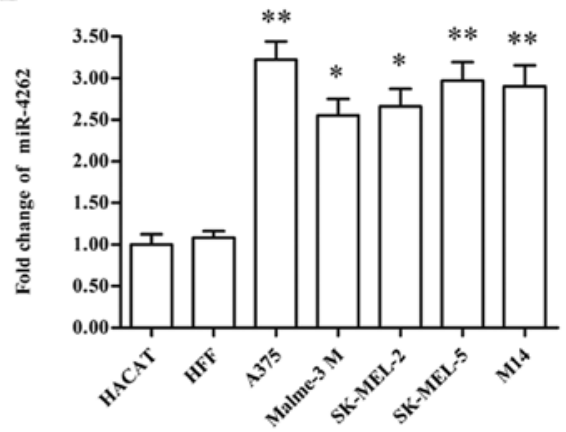

D
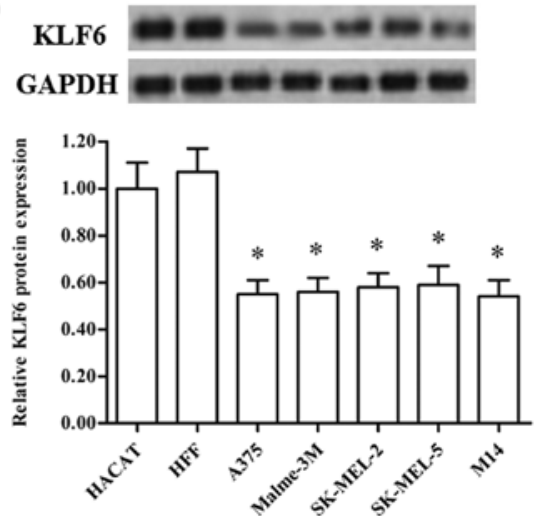

Figure 2. miR-4262 is markedly increased and KLF is decreased in human CMM cell lines. (A) The expression levels of pre-miR-4262 were markedly increased in human CMM cell lines. (B) The expression levels of mature miR-4262 were markedly increased in human CMM cell lines. (C) The levels of KLF6 mRNA were decreased in human CMM cell lines. (D) The levels of KLF6 protein were decreased in human CMM cell lines. Total RNA and total protein were respectively extracted from the HACAT normal human epithelial cell line, HFF precursor skin fibroblasts and 5 human CMM cell lines including A375, Malme-3M, SK-MEL-2, SK-MEL-5 and M14 when they grew to 100\% confluency. The expression levels of precursor miR-4262 (pre-miR-4262), mature miR-4262 and KLF6 mRNA were detected with real-time qPCR, and KLF6 protein expression was detected using western blotting. "P<0.05 vs. HACAT, ${ }^{* *} \mathrm{P}<0.01$ vs. HACAT. CMM, cutaneous malignant melanoma; KLF6, Kruppel-like 6.

A

\begin{tabular}{|c|c|}
\hline & \\
\hline & AAAAAA----GUA----GUUUGAAUGUUUUGUGUGUAAGGA \\
\hline & AAAAA-----GUA----GLUUGAAUGUULUUGUGUGUAA \\
\hline & -- GUA- \\
\hline & -- -GUUUGA \\
\hline & -- GUUUGA \\
\hline & ----GUC----GUUUG \\
\hline Pig & AAAAAAA-----AAACAGAGUUUGGAAUGUUCUGUGUGUGAG \\
\hline a mik-4L & $\begin{array}{l}\text { 3'- GUCCAUCAGACUUACAG -5' } \\
\text { Seed seguence }\end{array}$ \\
\hline
\end{tabular}

B

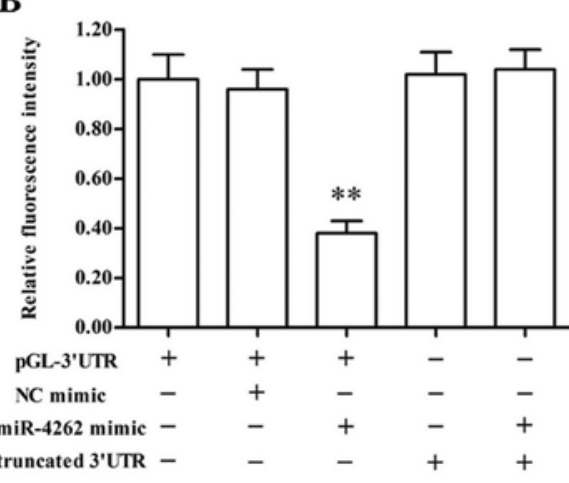

C
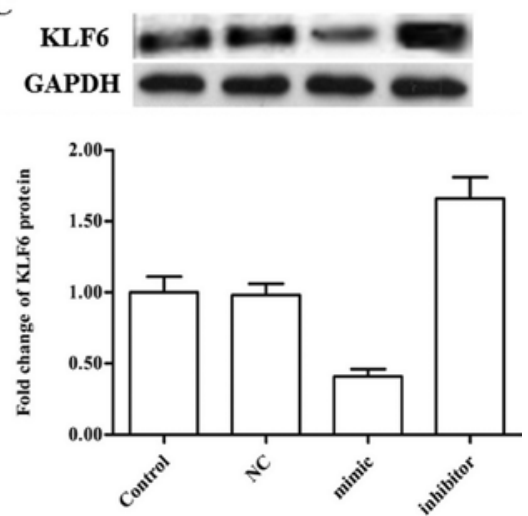

Figure 3. KLF6 is targeted by miR-4262 at the mRNA 3'UTR. (A) The seed sequence of miR-4262 was found to be completely matched with mRNA 3'UTR in various mammals. (B) KLF6 was identified as a target gene of miR-4262 by KLF6 mRNA 3'UTR luciferase reporter gene assay. The miR-4262 mimic was transfected alone or co-transfected with WT KLF6 mRNA 3'UTR reporter or the reporter with a truncated KLF6 mRNA 3'UTR lacking bases 390-420. After incubation for $72 \mathrm{~h}$, the luciferase intensity was detected with a luminometer. (C) KLF6 protein expression was sharply decreased by the miR-4262 mimic and increased by the miR-4262 inhibitor. The miR- 4262 mimic and inhibitor were transfected into A375 CMM cells at $80 \%$ confluency. After incubation for $72 \mathrm{~h}$, the KLF6 protein expression was detected using western blotting. ${ }^{* *} \mathrm{P}<0.01$ vs. psi-CHECK-WT 3'UTR, or control. KLF6, Kruppel-like 6 . 
A

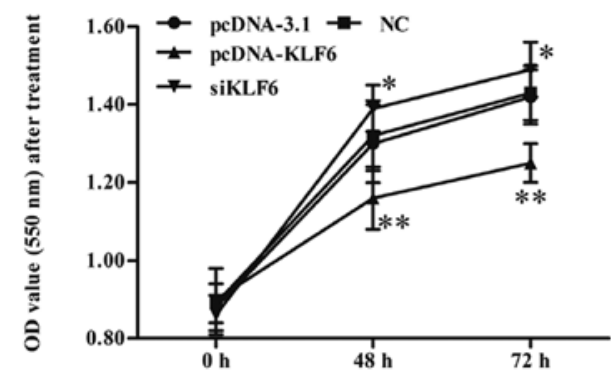

B

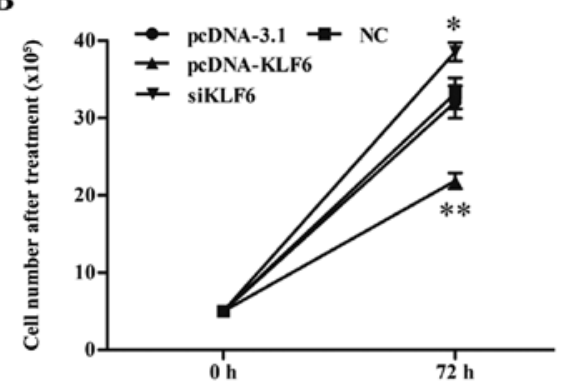

C
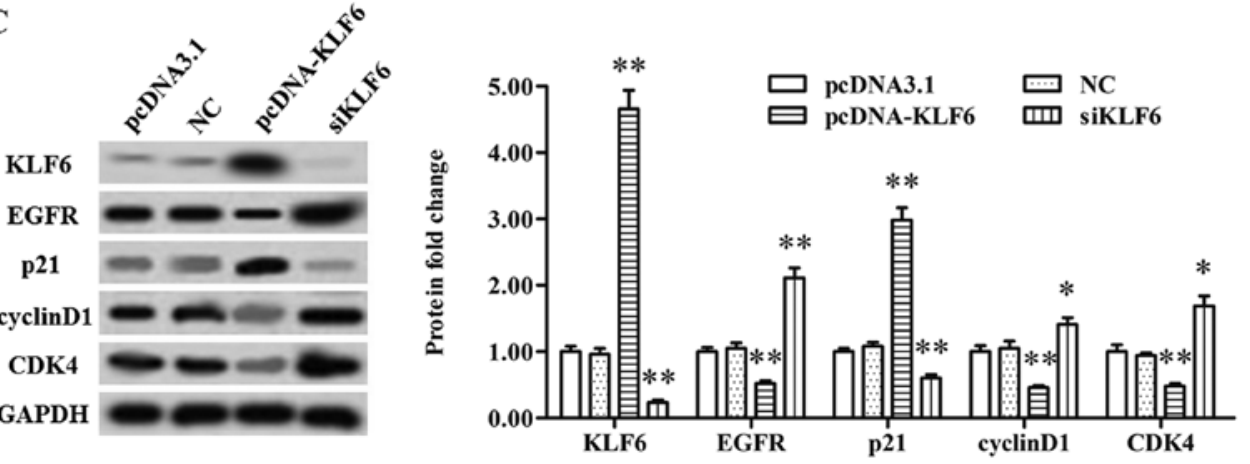

Figure 4. KLF6 suppresses A375 cell proliferation and the expression levels of EGFR and p21-mediated CDK4. (A) A375 cell proliferation was suppressed by KLF6 overexpression and increased by KLF6 knockdown as detected by MTT assay. (B) A375 cell proliferation was suppressed by KLF6 overexpression and increased by KLF6 knockdown as detected with CellTiter-Blue H assay. (C) KLF6 suppressed the protein levels of EGFR and p21-mediated CDK4. The pcDNA-KLF6, KLF6 siRNA, or relative negative control vector or siRNA were transfected into the CMM A375 cells at $80 \%$ confluency. After incubation for $72 \mathrm{~h}$, the protein expression levels of KLF6, EGFR, p21, cyclin D1 and CDK4 were detected using western blotting. " P $<0.05$, ** P $<0.01$. KLF6, Kruppel-like 6; EGFR, epidermal growth factor receptor; CDK4, cyclin-dependent kinase 4.

A

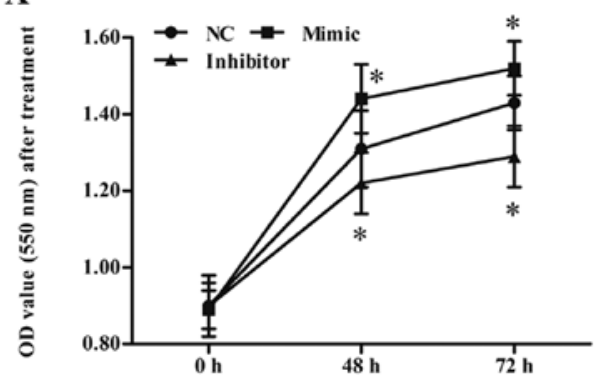

B

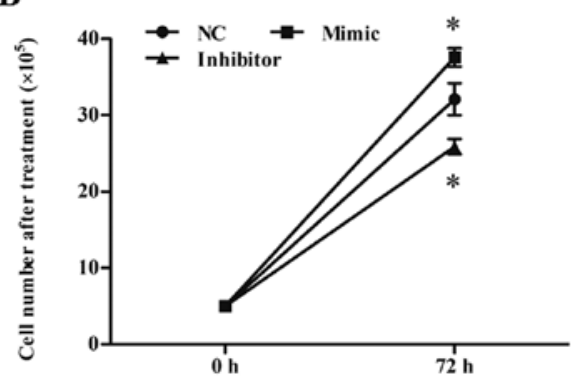

C
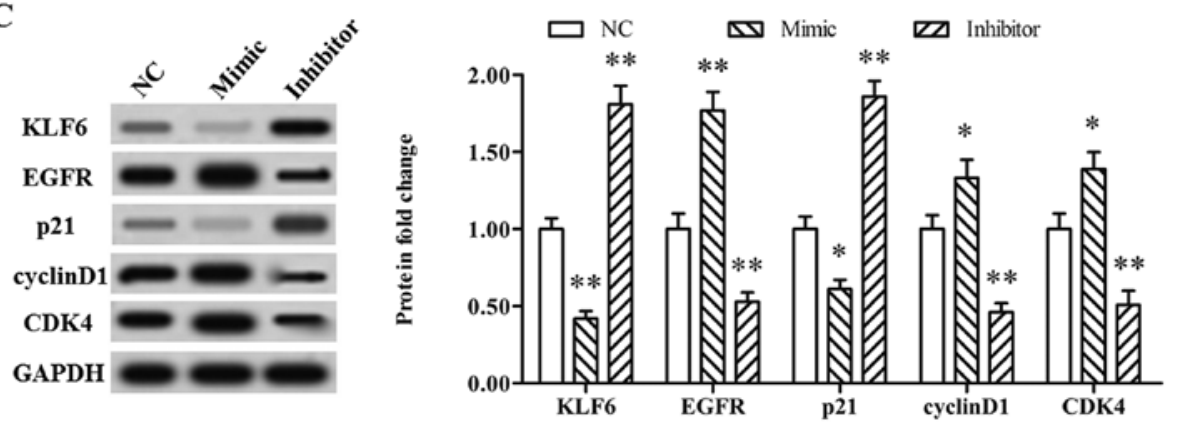

Figure 5. miR-4262 promotes A375 cell proliferation. (A) A375 cell proliferation was increased by the miR-4262 mimic and decreased by the miR-4262 inhibitor as detected by MTT assay. (B) A375 cell proliferation was increased by the miR- 4262 mimic and decreased by the miR- 4262 inhibitor as detected by CellTiter-Blue H assay. (C) miR-4262 increased the protein levels of EGFR and p21-mediated CDK4. The miR-4262 mimic and inhibitor were transfected into the CMM A375 cells at $80 \%$ confluency. After incubation for $72 \mathrm{~h}$, the protein expression levels of KLF6, EGFR, p21, cyclin D1 and CDK4 were detected using western blotting. ${ }^{*} \mathrm{P}<0.05,{ }^{* *} \mathrm{P}<0.01$. EGFR, epidermal growth factor receptor; CDK4, cyclin-dependent kinase 4; KLF6, Kruppel-like 6.

CellTiter-Blue $\mathrm{H}$ assays showed that A375 cell proliferation was markedly promoted by the miR-4262 mimic and reduced by the miR-4262 inhibitor (Fig. 5A and B). Moreover, the miR-4262 mimic significantly increased the level of EGFR, 
reduced that of $\mathrm{p} 21$, and caused an increase in the protein levels of cyclin D1 and CDK4 (Fig. 5C). The miR-4262 inhibitor had an opposite effect on protein expression (Fig. 5C). These results demonstrated that miR-4262 promoted the proliferation of CMM cells.

\section{Discussion}

miR-4262 was found in SOLiD ultra-deep small RNA sequencing of the Ago2 complex in human embryonic stem cells and neural precursors in 2009 (12), suggesting a potential regulatory role of miR-4262 in cell differentiation and proliferation. From then on, several studies have revealed that miR-4262 is dysregulated in several pathological processes, including tumor drug resistance, cancer metastasis and tissue fibrosis (14-16). In this study, we assessed the expression of miR-4262 in human CMM tissues and cell lines, as well as in matched normal epithelial tissues and cell lines. Our results showed that both pre- and mature miR-4262 were upregulated in the CMM tissues and cell lines, suggesting that miR-4262 may promote the progression of CMM.

As a newly discovered miRNA, the role of miR-4262 has been poorly studied. Here, we explored the effect of miR-4262 on the proliferation of CMM cell lines. Our data on miR-4262 overexpression and silencing both indicated that miR-4262 had a positive effect on the proliferation of CMM A375 cells. However, existing research results indicate that miR-4262 mainly plays a suppressive role in cancer progression. Song et al reported that the levels of miR-4262 were significantly decreased in osteosarcoma specimens and the 5-year survival of osteosarcoma patients with lower miR-4262 was reduced (17). Moreover, miR-4262 was found to target the 3'-UTR of osteopontin (OPN) mRNA and inhibit OPN-mediated cell invasion (17). A study on sequence matching between zinc finger E-box-binding homeobox 2 (ZEB2) and its targeting miRNA showed that miR-4262 potentially targets ZEB2, which was proven to be an E-cadherin repressor, and may play a negative role in larynx carcinoma progression (16). Another study on acute lung injury also indicated that miR-4262 targets the anti-apoptotic gene $\mathrm{Bcl}-2$ to induce apoptosis in pulmonary endothelial cells (18). We speculated that miR-4262 may play multiple roles in different biological processes. In fact, it is not a unique situation that miRNAs play versatile roles in different types of cells, or even in the same type of cells under different conditions. A typical example is miR-210, an extensively investigated miRNA in cancer biology that is regarded as a signature of hypoxia, that functions either as a tumor promoter or a tumor suppressor, and can be a positive or negative prognostic biomarker (19-22).

It has been proven that the function of KLF6 is determined by the length of its splice variants (SVs). KLF6-full-length (KLF6-FL or KLF6) protein is downregulated and functions as a tumor suppressor in cancers, while truncated KLF6-SVs are usually expressed at higher levels in tumors and drive cancer growth and metastasis $(5,23,24)$. The length of KLF6 transcripts has been associated with the DNA methylation level in the KLF6 promoter region (25). Treatment with the demethylating agent 5-aza-2'-deoxycytidine caused upregulation of KLF6 expression and a relative decrease in KLF6 SVs (26). As a tumor growth suppressor, KLF6 promotes the transactivation of the anti-tumorigenic gene p21 to suppress the cyclin D1/CDK-mediated cell cycle (27). KLF6 was also found to negatively regulate EGFR signaling in both cell culture and in vivo models and was proposed as an anti-EGFR-based therapy for the treatment of metastatic carcinomas (28). However, the exact role of KLF6 in human CMM progression has not been well studied. In this study, we reported that KLF6 was significantly downregulated in CMM cell lines and played a negative regulatory role in the proliferation of CMM cells. Moreover, KLF6 overexpression in CMM cells caused downregulation of EGFR and a robust increase in p21 expression. In contrast, knockdown of KLF6 increased EGFR and reduced p21 expression. KLF6 had a negative effect on CMM cell proliferation through inactivation of the EGFR/PI3K/Akt and p21-mediated cyclin D1/CDK pathways.

In conclusion, miR-4262 was found to be upregulated in CMM tissues and cell lines compared with these levels in matched normal epithelial tissues and normal cell lines. miR-4262 promoted the proliferation of CMM cells through targeting of the tumor suppressor KLF6.

\section{Acknowledgements}

This study was supported by the Fundamental Research Funds for the Central Universities.

\section{References}

1. Moan JE, Baturaite Z, Dahlback A and Porojnicu AC: Ultraviolet radiation and cutaneous malignant melanoma. Adv Exp Med Biol 810: 359-374, 2014

2. Law MH, Bishop DT, Lee JE, Brossard M, Martin NG, Moses EK, Song F, Barrett JH, Kumar R, Easton DF, et al; GenoMEL Consortium; Essen-Heidelberg Investigators; SDH Study Group; Q-MEGA and QTWIN Investigators; AMFS Investigators; ATHENS Melanoma Study Group: Genome-wide meta-analysis identifies five new susceptibility loci for cutaneous malignant melanoma. Nat Genet 47: 987-995, 2015.

3. Emri G, Emri E, Boros G, Hegedús C, Janka E, Gellén E and Remenyik E: Skin carcinogenesis: the pathogenetic and therapeutic role of zinc. J Metallomics Nanotechnol 2: 19-26, 2015.

4. Atkins GB and Jain MK: Role of Krüppel-like transcription factors in endothelial biology. Circ Res 100: 1686-1695, 2007.

5. DiFeo A, Martignetti JA and Narla G: The role of KLF6 and its splice variants in cancer therapy. Drug Resist Updat 12: 1-7, 2009.

6. Narla G, DiFeo A, Yao S, Banno A, Hod E, Reeves HL, Qiao RF, Camacho-Vanegas O, Levine A, Kirschenbaum A, et al: Targeted inhibition of the KLF6 splice variant, KLF6 SV1, suppresses prostate cancer cell growth and spread. Cancer Res 65: 5761-5768, 2005.

7. Reeves HL, Narla G, Ogunbiyi O, Haq AI, Katz A, Benzeno S, Hod E, Harpaz N, Goldberg S and Tal-Kremer S: Kruppel-like factor 6 (KLF6) is a tumor-suppressor gene frequently inactivated in colorectal cancer. Gastroenterology 126: 1090-1103, 2004.

8. Kremer-Tal S, Reeves HL, Narla G, Thung SN, Schwartz M, Difeo A, Katz A, Bruix J, Bioulac-Sage P, Martignetti JA, et al: Frequent inactivation of the tumor suppressor Kruppel-like factor 6 (KLF6) in hepatocellular carcinoma. Hepatology 40: 1047-1052, 2004.

9. Ebrahimi A, Nodushan SMHT, Mousavian A, Mokarizadeh A, Abbasi M, Yahaghi E and Rasaei SM: Diagnostic and prognostic potentials of KLF6 and HER3 expression alterations in cutaneous malignant melanoma. Tumour Biol: Oct 16, 2015 (Epub ahead of print).

10. Cai D, Zhao J and Sun Q: Kruppel-like factor 6 in the progression and prognosis of malignant melanoma. J Int Med Res 42: 184-190, 2014. 
11. Lin WM, Baker AC, Beroukhim R, Winckler W, Feng W, Marmion JM, Laine E, Greulich $\mathrm{H}$, Tseng $\mathrm{H}$, Gates $\mathrm{C}$, et al: Modeling genomic diversity and tumor dependency in malignant melanoma. Cancer Res 68: 664-673, 2008.

12. Goff LA, Davila J, Swerdel MR, Moore JC, Cohen RI, Wu H, Sun YE and Hart RP: Ago2 immunoprecipitation identifies predicted microRNAs in human embryonic stem cells and neural precursors. PLoS One 4: e7192, 2009.

13. Rönnau CG, Verhaegh GW, Luna-Velez MV and Schalken JA: Noncoding RNAs as novel biomarkers in prostate cancer. Biomed Res Int 2014: 591703, 2014.

14. Pouliot LM, Shen D-W, Suzuki T, Hall MD and Gottesman MM: Contributions of microRNA dysregulation to cisplatin resistance in adenocarcinoma cells. Exp Cell Res 319: 566-574, 2013.

15. Jing L, Jin $\mathrm{C}$, Lu Y, Huo $\mathrm{P}$, Zhou L, Wang $\mathrm{Y}$ and Tian $\mathrm{Y}$ : Investigation of microRNA expression profiles associated with human alcoholic cardiomyopathy. Cardiology 130:223-233, 2015.

16. Gao S, Wang J, Xie J, Zhang T and Dong P: Role of miR-138 in the regulation of larynx carcinoma cell metastases. Tumour Biol: Oct 24, 2015 (Epub ahead of print).

17. Song K, Liu N, Yang Y and Qiu X: Regulation of osteosarcoma cell invasion through osteopontin modification by miR-4262. Tumour Biol 37: 6493-6499, 2015.

18. Bao H, Gao F, Xie G and Liu Z: Angiotensin-converting enzyme 2 inhibits apoptosis of pulmonary endothelial cells during acute lung injury through suppressing miR-4262. Cell Physiol Biochem 37: 759-767, 2015.

19. Huang X, Le Q-T and Giaccia AJ: MiR-210 - micromanager of the hypoxia pathway. Trends Mol Med 16: 230-237, 2010.

20. Chan YC, Banerjee J, Choi SY and Sen CK: miR-210: The master hypoxamir. Microcirculation 19: 215-223, 2012.

21. Wang H, Flach H, Onizawa M, Wei L, McManus MT and Weiss A: Negative regulation of Hifla expression and TH17 differentiation by the hypoxia-regulated microRNA miR-210. Nat Immunol 15: 393-401, 2014
22. Qin Q, Furong W and Baosheng L: Multiple functions of hypoxia-regulated miR-210 in cancer. J Exp Clin Cancer Res 33: $50,2014$.

23. Hatami R, Sieuwerts AM, Izadmehr S, Yao Z, Qiao RF, Papa L, Look MP, Smid M, Ohlssen J, Levine AC, et al: KLF6-SV1 drives breast cancer metastasis and is associated with poor survival. Sci Transl Med 5: 169ra12, 2013.

24. Zhenzhen Z, De'an T, Limin X, Wei Y and Min L: New candidate tumor-suppressor gene KLF6 and its splice variant KLF6 SV2 counterbalancing expression in primary hepatocarcinoma. Hepatogastroenterology 59: 473-476, 2012.

25. Andreoli V, Gehrau RC and Bocco JL: Biology of Krüppel-like factor 6 transcriptional regulator in cell life and death. IUBMB Life 62: 896-905, 2010.

26. Chen $\mathrm{CH}$, Huang $\mathrm{PH}$, Chu $\mathrm{PC}$, Chen $\mathrm{MC}$, Chou $\mathrm{CC}$, Wang D, Kulp SK, Teng CM, Wang Q and Chen CS: Energy restriction-mimetic agents induce apoptosis in prostate cancer cells in part through epigenetic activation of KLF6 tumor suppressor gene expression. J Biol Chem 286: 9968-9976, 2011.

27. Lang UE, Kocabayoglu P, Cheng GZ, Ghiassi-Nejad Z Muñoz U, Vetter D, Eckstein DA, Hannivoort RA, Walsh MJ and Friedman SL: GSK3 $\beta$ phosphorylation of the KLF6 tumor suppressor promotes its transactivation of $\mathrm{p} 21$. Oncogene 32 : 4557-4564, 2013.

28. Sangodkar J, Dhawan NS, Melville H, Singh VJ, Yuan E, Rana H, Izadmehr S, Farrington C, Mazhar S, Katz S, et al: Targeting the FOXO1/KLF6 axis regulates EGFR signaling and treatment response. J Clin Invest 122: 2637-2651, 2012. 